\title{
EVALUATION OF LIFESTYLE CHANGES IN PATIENTS DIAGNOSED WITH LUNG CANCER
}

\section{Introduction:}

Lung cancer remains the main cause for morbidity and mortality among all cancer types worldwide. Both the disease and its treatment cause symptoms which undermine patients' quality of life affecting their physical, social and psychological well-being.

\section{Objectives}

Evaluation of the impact of lung cancer disease on QOL

\section{Methods}

The sample of the study consisted of 100 lung cancer patients attending a Greek oncology clinic. QLQ-C30 \& QoL-LC13 questionnaires were used.

\section{Results}

Cognitive Function was at $65.93 \%$, Social Function at $67.50 \%$, Physical Function at $65.93 \%$ with the General Health at $60.83 \%$. Financial Difficulties were at $41.0 \%$. Most common symptoms were fatigue, constipation, insomnia, dyspnea and alopecia. Pain was found at $14.63 \%$ with those under medication scoring less in the General Health and Physical Function variable. Diarrhea was significantly higher among patients without children $(p=0.038)$ and insomnia among the working patients $(p=0.043)$

\section{Conclusions}

Factors that affects the QOL of patients have been found to be marital status, existence of children, work and pain medication. Gender, age, educatio were not found to be factors influencing the quality of life.

Keywords: quality of life, lung cancer, life style changes

Co-authors

M. Kiagia 1, E. Koutsikouri 1, A. Marioli 1, E. Dalakou 1, G. Tourkantonis 1, I. Karambelas 1, K. Syrigos 1

1 Sotiria General Hospital, Oncology Unit GPP, Athens, Greece 